\title{
Intraoperative Neurophysiological Monitoring for Spinal Cord Tumor Surgery: Comparison of Motor and Somatosensory Evoked Potentials According to Tumor Types
}

\author{
Taeha Park, MD, Jinyoung Park, MD, Yoon Ghil Park, MD, PhD, Joowon Lee, MD \\ Department of Rehabilitation Medicine and Rehabilitation Institute of Neuromuscular Disease, \\ Yonsei University College of Medicine, Seoul, Korea
}

\begin{abstract}
Objective To identify which combination of motor evoked potentials (MEPs) and somatosensory evoked potentials (SEPs) is most reliable for postoperative motor deterioration during spinal cord tumor surgery, according to anatomical and pathologic type.

Methods MEPs and SEPs were monitored in patients who underwent spinal cord tumor surgery between November 2012 and August 2016. Muscle strength was examined in all patients before surgery, within 48 hours postoperatively and 4 weeks later. We analyzed sensitivity, specificity, positive and negative predictive values of each significant change in SEPs and MEPs.

Results The overall sensitivity and specificity of SEPs or MEPs were $100 \%$ and $61.3 \%$, respectively. The intraoperative MEP monitoring alone showed both higher sensitivity (67.9\%) and specificity (83.2\%) than SEP monitoring alone for postoperative motor deterioration. Two patients with persistent motor deterioration had significant changes only in SEPs. There are no significant differences in reliabilities between anatomical types, except with hemangioma, where SEPs were more specific than MEPs for postoperative motor deterioration. Both overall positive and negative predictive values of MEPs were higher than the predictive values of SEPs. However, the positive predictive value was higher by the dual monitoring of MEPs and SEPs, compared to MEPs alone.

Conclusion For spinal cord tumor surgery, combined MEP and SEP monitoring showed the highest sensitivity for the postoperative motor deterioration. Although MEPs are more specific than SEPs in most types of spinal cord tumor surgery, SEPs should still be monitored, especially in hemangioma surgery.
\end{abstract}

Keywords Spinal cord neoplasm, Intraoperative neurophysiological monitoring, Motor evoked potentials, Somatosensory evoked potentials, Postoperative complications

Received October 13, 2016; Accepted October 24, 2016

Corresponding author: Joowon Lee

Department of Rehabilitation Medicine and Rehabilitation Institute of Neuromuscular Disease, Gangnam Severance Hospital, Yonsei University College of Medicine, 211 Eonju-ro, Gangnam-gu, Seoul 06273, Korea. Tel: +82-2-2019-3492, Fax: +82-2-3463-7585, E-mail: HANLEZZ@yuhs.ac ORCID: Taeha Park (http://orcid.org/0000-0003-3316-5330); Jinyoung Park (http://orcid.org/0000-0003-4042-9779); Yoon Ghil Park (http://orcid. org/0000-0001-9054-5300); Joowon Lee (http://orcid.org/0000-0003-0342-3159).

@ This is an open-access article distributed under the terms of the Creative Commons Attribution Non-Commercial License (http://creativecommons.org/ licenses/by-nc/4.0) which permits unrestricted noncommercial use, distribution, and reproduction in any medium, provided the original work is properly cited. Copyright $\odot 2017$ by Korean Academy of Rehabilitation Medicine 


\section{INTRODUCTION}

Improvements in surgical techniques increasingly lead to more aggressive tumor resections. However, even for the most skillful surgeons, spine surgery has an inherent risk of postoperative neurological deterioration [1]. Intraoperative neurophysiologic monitoring (IONM) enables the surgeon to proceed with more aggressive maneuvers for near total removal of the tumor [2].

There have been various studies on effective monitoring, based on the clinical importance of IONM for intramedullary spinal cord tumor surgery. However, a specific guideline on IONM techniques has yet to be established. Recently, Scibilia et al. [3] reviewed several previous studies on different combinations of IONM techniques (such as somatosensory evoked potentials [SEPs], motor evoked potentials [MEPs], direct wave, free-running electromyography), and emphasized the multimodal IONM for safe spinal cord tumor surgery. There are controversies concerning certain IONM combination techniques, but they found combined motor and somatosensory evoked potential monitoring is most commonly used for spinal cord tumor surgeries.

A follow-up study compared certain pathologic types of spinal cord tumors to identify which type easily affects the IONM alarm criteria [4]. Considering the signal pathways of MEPs and SEPs, the vulnerability to the tracts would differ by anatomical and pathologic types.

Theoretically, using combined MEPs and SEPs for all spinal cord tumor surgeries would be the most sensitive for postoperative neurologic complications. However, considering cost-effectiveness and time constraints, establishing a standard guideline would be helpful. Such a guideline should suggest each combination of evoked potentials, according to the different types of spinal cord tumors.

There have been many studies on IONM and spine surgery, but studies particularly focusing on spinal cord tumor cases are not common. Furthermore, very few studies have categorized spinal cord tumors by anatomical and pathologic type, or examined the reliability of IONM for each spinal cord tumor type.

To fill this gap in research, our study analyzes IONM cases of spinal cord tumor resection and identifies which combination of MEPs and SEPs is most reliable during spinal cord tumor surgery. We identify which combina- tion is the most reliable by anatomical and pathologic type, which thereby enables us to better predict the postoperative motor deterioration.

\section{MATERIALS AND METHODS}

\section{Subjects}

Between November 2012 and August 2016, we monitored intraoperative MEPs or SEPs during 123 spinal cord tumor operations. Extensively experienced spinal surgeons performed all of these operations. We conducted a prospective analysis on the patient data, which included IONM records, medical records, operative narratives, anesthesia records, and outpatient clinical records. Preoperatively, 59 patients were neurologically intact and 64 patients had motor deteriorations. Table 1 lists the baseline characteristics of the patients.

\section{Anesthesia}

Rocuronium bromide Esmeron 50-150 mg (Han Wha Pharma Co. Ltd., Seoul, Korea) was administered intravenously as a short-acting muscle relaxant to facilitate endotracheal intubation. No paralytic agents were subsequently administered.

General anesthesia was induced by total intravenous anesthesia with remifentanil Ultiva (GSK, Middlesex, UK), propofol Fresofol (Fresenius Kabi, Seoul, Korea), or midazolam Vascam (Hana Pharm, Seoul, Korea) were used in several combinations to initiate and maintain the general anesthesia. During anesthesia, body temperature, direct radial artery pressure, pulse rate, oxygen saturation, and end-tidal carbon dioxide concentration were continuously monitored. All patients were kept normothermic and normotensive.

\section{IONM monitoring techniques}

A single technician performed IONM using the Cascade (Cadwell Industries Inc., Kennewick, WA, USA). In 110 out of 123 patients, both MEPs and SEPs were monitored. In the other 13 patients, only MEPs were monitored. Median SEPs were monitored in 38 patients, and tibial SEPs were monitored in 104 cases.

\section{Somatosensory evoked potentials}

SEPs were elicited by electrical stimulation of the median nerve at the wrist and the posterior tibial nerve at the 
Table 1. Baseline demographic and clinical characteristics of patients

\begin{tabular}{|c|c|c|c|c|}
\hline Variable & $\begin{array}{c}\text { Total } \\
(n=123)\end{array}$ & $\begin{array}{l}\text { Group A } \\
(\mathbf{n}=109)\end{array}$ & $\begin{array}{c}\text { Group B } \\
(n=14)\end{array}$ & p-value \\
\hline Age (yr) & $48.3(6-87)$ & $48.6(6-87)$ & $45.3(24-75)$ & 0.46 \\
\hline Sex & & & & 0.58 \\
\hline Male & $53(43.1)$ & $46(42.2)$ & $7(50.0)$ & \\
\hline Female & $70(56.9)$ & $63(57.8)$ & $7(50.0)$ & \\
\hline \multicolumn{5}{|c|}{ Preoperative motor score } \\
\hline Preoperative & $95.0(62-100)$ & $95.3(62-100)$ & $91.7(70-100)$ & \\
\hline Postoperative & $93.4(60-100)$ & $95.4(63-100)$ & $79.5(60-94)$ & \\
\hline \multicolumn{5}{|l|}{ Anatomical type } \\
\hline IDEM & $79(64.2)$ & $78(71.6)$ & $1(7.1)$ & \\
\hline IM & $31(25.2)$ & $19(17.4)$ & $12(85.7)$ & \\
\hline $\mathrm{ED}$ & $11(8.9)$ & $10(9.2)$ & $1(7.1)$ & \\
\hline IDEM and ED & $2(1.6)$ & $2(1.8)$ & $0(0.0)$ & \\
\hline \multicolumn{5}{|l|}{ Pathological type } \\
\hline Schwannoma & $51(41.5)$ & $50(45.9)$ & $1(7.1)$ & \\
\hline Meningioma & $23(18.7)$ & $23(21.1)$ & $0(0.0)$ & \\
\hline Ependymoma & $15(12.2)$ & $6(5.5)$ & $9(64.3)$ & \\
\hline Hemangioma & $6(4.9)$ & $4(3.7)$ & $2(14.3)$ & \\
\hline Neurofibroma & $5(4.1)$ & $5(4.6)$ & $0(0.0)$ & \\
\hline Cystic lesion & $5(4.1)$ & $4(3.7)$ & $1(7.1)$ & \\
\hline Metastasis & $4(3.3)$ & $4(3.7)$ & $0(0.0)$ & \\
\hline Others ${ }^{\text {a) }}$ & $14(11.4)$ & $13(11.9)$ & $1(7.1)$ & \\
\hline
\end{tabular}

Values are presented as mean (range) or number (\%).

IDEM, intradural extramedullary; IM, intramedullary; ED, extradural; Group A, postoperatively motor intact; Group B, postoperatively motor deterioration.

${ }^{\text {a) }}$ Others were 1 astrocytoma, 2 multiple myelomas, 1 malignant melanoma, 1 paraganglioma, 1 small round cell tumor, 1 osteochondroma, 1 chondrosarcoma, 1 giant cell tumor, 1 neurothekeoma, and 2 lipomas.

ankle (intensity $40 \mathrm{~mA}$, duration $0.2 \mathrm{~ms}$, with a repetition rate of $5 \mathrm{~Hz}$ ). We recorded SEPs from needle electrodes placed on the scalp at C3 (right median nerve), C4 (left median nerve), and $\mathrm{Cz}$ (right or left tibial nerve) referenced to Fpz according to the 10-20 international electroencephalography (EEG) system.

\section{Motor evoked potentials}

We obtained motor evoked potentials by multipulse transcranial electric stimulations using the Cascade electrical stimulator (Cadwell Industries Inc.). We recorded transcranial electric motor evoked potentials bilaterally from the abductor pollicis brevis muscles in upper extremities, and tibialis anterior muscles in the lower extremities using a pair of needle electrodes inserted $3 \mathrm{~cm}$ apart in each muscle. Needle electrodes delivered short trains of 6 square-wave stimuli of $0.5 \mathrm{~ms}$ duration, with an interstimulus interval of $5 \mathrm{~ms}$. The needles delivered up to $2 \mathrm{~Hz}$ of repetition rate and were placed at $\mathrm{Cl}$ and C2, according to the 10-20 international EEG system. A $\mathrm{C} 1 / \mathrm{C} 2$ montage is preferable for right-extremity MEPs, while $\mathrm{C} 2 / \mathrm{C} 1$ is preferentially used to elicit left-extremity MEPs. To elicit lower-extremity MEPs, we used a $\mathrm{Cz} / \mathrm{Fz}$ montage, which produces less intense muscle twitching. We gradually escalated the intensity of the stimulus by 50 $\mathrm{mV}$ increments (from $50 \mathrm{mV}$ to a maximum of $400 \mathrm{mV}$ ) until MEP amplitudes were maximized above a minimum of $10 \mathrm{mV}$.

\section{Alarm criteria for evoked potentials}

We performed neurophysiological monitoring throughout the surgical procedures. We obtained baseline EPs 
after at least 60 minutes after intubation and awaited the muscle relaxant effect to fade.

We continuously monitored values of N20 latencies of median SEPs, P37 latencies of tibial SEPs, and peak-topeak amplitudes of MEPs. We considered a more than $50 \%$ reduction in amplitude, compared to the baseline MEPs, as a significant change $[5,6]$. We defined the prolongation of N20 or P37 latencies of more than 10\% from the baseline SEPs as a significant change [7]. If any significant EP changes occurred, the surgeons were promptly informed and the surgical procedures were stopped temporarily until the values of EPs returned to normal. However, if no signal reversal occurred even after the surgical correction or after removing the spinal implant placed immediately prior to signal change, surgeons considered procedure cessation.

\section{Neurologic examination}

We evaluated the motor status of each patient before surgery, 48 hours after surgery, and then 4 weeks later. We measured the strengths of 10 key muscles of the International Standards for Neurological Classification of Spinal Cord Injury. We evaluated muscle strength using the Medical Research Council (MRC) scale range of 0 to 5 . The total score ranged from 0 to 50 points on each side. We considered any motor reduction scoring with 1 point or more, compared to the preoperative evaluation, as 'postoperative neurologic motor deterioration.' We considered any postoperative weakness observed within 48 hours after surgery, which subsequently recovered after 4 weeks, as a 'transient motor deterioration.' If the decreased motor score at 48 hours did not recover in 4 weeks, we considered the patient to have 'persistent postoperative motor deterioration.'

We conducted statistical analyses to reveal the correlations between the results of intraoperative changes of SEPs or MEPs and the motor status (intact, transient or persistent motor deterioration). We conducted the analyses according to each anatomical or pathologic type of spinal cord tumor.

\section{Statistical analysis}

We used an independent t-test to compare the mean age, height and sex between the patients with motor deterioration (transient and persistent) and the patients without motor deterioration. We calculated the sensitiv- ity, specificity, positive and negative predictive values in each type of spinal cord tumor by anatomical lesion and pathology. We analyzed this data using SPSS ver. 20.1 (IBM, Armonk, NY, USA), with p-values of $<0.05$ considered statistically significant.

\section{RESULTS}

\section{Baseline characteristics of the subjects}

We enrolled a total of 123 spinal cord tumor patients in this study. Fourteen patients had shown motor deterioration, 8 had motor improvement, 101 had no motor change postoperatively, and 9 of postoperative motor deterioration patients had preoperative motor deterioration already (Table 2).

Table 1 displays the baseline demographic and clinical characteristics of the population. There were 53 male patients $(43.1 \%)$ and 70 female patients $(56.9 \%)$. The mean age of the patients was 48.3 years. Table 1 also compares the group that was neurologically intact postoperatively (group A) to the group with postoperative motor deteriorations (group B). There were no statistically significant differences in age and sex.

We classified the types of tumors by anatomy; there were 79 intradural extramedullary (IDEM) tumors; 31 intramedullary (IM) tumors; 11 extradural (ED) tumors; and 2 cases of IDEM combined with ED tumors. We also categorized the patients according to pathology: there were 51 schwannomas, 25 meningiomas, 15 ependymomas, 6 hemangiomas, 5 neurofibromas, 5 cystic lesions, 4 metastatic tumors, and 14 'others' (Table 1).

\section{Postoperative motor deteriorations}

Among 123 patients, 109 patients showed no postoperative motor deterioration. Of the 14 patients with postoperative motor deterioration, 7 patients had significant

Table 2. Preoperative and postoperative motor change of patients

\begin{tabular}{|c|c|c|c|}
\hline & $\begin{array}{c}\text { Postoperative } \\
\text { motor } \\
\text { improvement } \\
(n=8)\end{array}$ & $\begin{array}{c}\text { Postoperative } \\
\text { no motor } \\
\text { change } \\
(n=101)\end{array}$ & $\begin{array}{c}\text { Postoperative } \\
\text { motor } \\
\text { deterioration } \\
(n=14)\end{array}$ \\
\hline \multicolumn{4}{|c|}{ Preoperative motor score } \\
\hline $100(n=59)$ & 0 & 54 & 5 \\
\hline$<100(\mathrm{n}=64)$ & 8 & 47 & 9 \\
\hline
\end{tabular}


changes in both MEPs and SEPs, 5 patients had only MEP changes, and 2 patients had only SEP changes (Table 3 ).

Ten patients showed persistent motor deteriorations. We observed both MEP and SEP changes in 4 patients, MEP changes alone in 4 patients, and SEP changes alone in 2 patients (Fig. 1).

\section{Combinations of intraoperative neurophysiologic monitoring}

We monitored all patients in this study with intraoperative MEPs or SEPs. Both intraoperative MEPs and SEPs had no significant changes during surgery in 73 patients (59.4\%). In 15 cases (12.2\%), only amplitude of MEPs significantly decreased without significant changes of SEPs. In 23 cases, only latencies of SEPs were significantly prolonged (18.7\%). We observed both significant changes in MEPs and SEPs in 12 cases (9.8\%).

\section{Reliability of intraoperative neurophysiologic monitoring}

After comparing the patients' motor status at 48 hours and at one month after surgery with the intraoperative EPs data, we calculated four measurements of reliability for each category of tumor by anatomy or pathology. Table 4 illustrates these calculations.

Overall, for MEP changes, sensitivity was $67.9 \%$, speci- ficity was $83.2 \%$, positive predictive value was $48.7 \%$, and negative predictive value was $91.7 \%$. For SEP changes, sensitivity was $57.1 \%$, specificity was $73.9 \%$, positive predictive value was $34.0 \%$, and negative predictive value was $\mathbf{8 8 . 0 \%}$. For both MEP and SEP changes, sensitivity was $25.0 \%$, specificity was $95.8 \%$, positive predictive value was $58.3 \%$, and negative predictive value was $84.4 \%$. For MEP or SEP changes, sensitivity was $100 \%$, specificity was $61.3 \%$, positive predictive value was $37.8 \%$, and negative predictive value was $100 \%$ (Table 4 ).

As shown by this data, both overall positive and negative predictive values of MEPs were higher than the predictive values of SEPs. However, the positive predictive value was higher by the dual monitoring of MEPs and SEPs compared to MEPs alone.

\section{Analysis by anatomical type}

We calculated the reliabilities by anatomical type. Table 5 illustrates these calculations.

In ED tumors, for MEP changes, sensitivity was $66.7 \%$ and specificity was $100 \%$. For SEP changes, sensitivity was $66.7 \%$ and specificity was $80.0 \%$. There was no significant difference between sensitivity and specificity of MEP and SEP. However, in IM tumors, for MEP changes, sensitivity was $70.8 \%$ and specificity was $81.0 \%$. For SEP changes, sensitivity were $54.2 \%$, specificity was $71.4 \%$.

Table 3. Patients' data with postoperative motor deteriorations after spinal cord tumor surgery

\begin{tabular}{cclcllc}
\hline $\begin{array}{c}\text { Case } \\
\text { no. }\end{array}$ & $\begin{array}{c}\text { Age (yr)/ } \\
\text { sex }\end{array}$ & $\begin{array}{c}\text { Pathologic } \\
\text { diagnosis }\end{array}$ & $\begin{array}{c}\text { Anatomical } \\
\text { type }\end{array}$ & $\begin{array}{c}\text { Level } \\
\text { of lesion }\end{array}$ & \multicolumn{1}{c}{ EPs change } & $\begin{array}{c}\text { Motor } \\
\text { deterioration }\end{array}$ \\
\hline 1 & $75 / \mathrm{F}$ & Schwannoma & IDEM & C3-4 & SEPs & Persistent \\
\hline 2 & $24 / \mathrm{M}$ & Anaplastic astrocytoma & IM & C2-T1 & SEPs & Persistent \\
\hline 3 & $61 / \mathrm{M}$ & Ependymoma & IM & C5-7 & MEPs & Persistent \\
\hline 4 & $54 / \mathrm{M}$ & Ependymoma & IM & C2-4 & MEPs and SEPs & Persistent \\
\hline 5 & $34 / \mathrm{M}$ & Ependymoma & ED & T11-12 & MEPs and SEPs & Persistent \\
\hline 6 & $44 / \mathrm{F}$ & Ependymoma & IM & C2-T2 & MEPs & Persistent \\
\hline 7 & $56 / \mathrm{F}$ & Cavernous hemangioma & IM & T11-12 & MEPs and SEPs & Persistent \\
\hline 8 & $43 / \mathrm{M}$ & Cavernous hemangioma & IM & T6 & MEPs and SEPs & Persistent \\
\hline 9 & $58 / \mathrm{M}$ & Ependymoma & IM & C2-7 & MEPs & Persistent \\
\hline 10 & $24 / F$ & Ependymoma & IM & T10-11 & MEPs & Persistent \\
\hline 11 & $44 / F$ & Ependymoma & IM & C2-T4 & MEPs and SEPs & Transient \\
\hline 12 & $49 / F$ & Ependymoma & IM & T7-8 & MEPs and SEPs & Transient \\
\hline 13 & $39 / \mathrm{M}$ & Ependymoma & IM & C4-7 & MEPs & Transient \\
\hline 14 & $29 / F$ & Cystic glioma & IM & C2-5 & MEPs and SEPs & Transient \\
\hline
\end{tabular}

MEPs, motor evoked potentials; SEPs, somatosensory evoked potentials; IDEM, intradural extramedullary; IM, intramedullary; ED, extradural. 


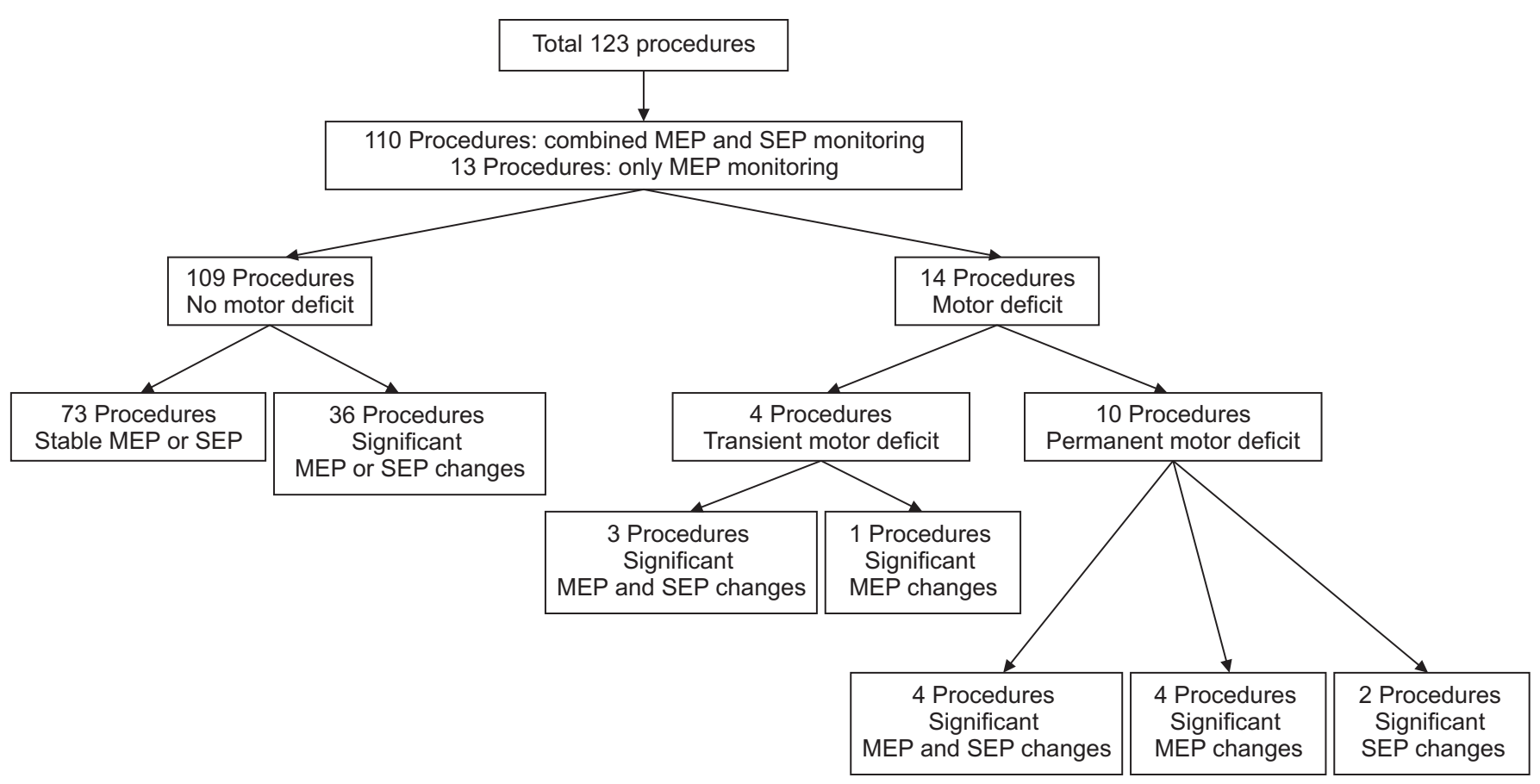

Fig. 1. Intraoperative neurophysiologic monitoring and clinical outcome in spinal cord tumor surgery. MEP, motor evoked potential; SEP, somatosensory evoked potential.

Table 4. Overall reliabilities of each combination of intraoperative MEPs and SEPs for the postoperative motor deterioration

\begin{tabular}{lcccc}
\hline Significant changes & Sensitivity (\%) & Specificity (\%) & PPV (\%) & NPV (\%) \\
\hline MEPs only & 67.9 & 83.2 & 48.7 & 91.7 \\
SEPs only & 57.1 & 73.9 & 34.0 & 88.0 \\
Both MEPs and SEPs & 25.0 & 95.8 & 58.3 & 84.4 \\
\hline Either MEPs or SEPs & 100 & 61.3 & 37.8 & 100 \\
\hline
\end{tabular}

MEPs, motor evoked potentials; SEPs somatosensory evoked potentials; PPV, positive predictive value; NPV, negative predictive value.

We can conclude from this data the specificity and sensitivity of MEPs for the postoperative motor deterioration was superior to SEPs in intramedullary tumors. Both positive and negative predictive values of MEPs in intramedullary tumor surgery were higher than with SEPs (Table 5).

\section{Analysis by pathologic type}

The specificity of MEPs for the postoperative motor deterioration was superior in schwannoma and meningioma. In ependymoma, the sensitivity of MEPs was superior to SEPs, but the specificities were the same. However, the specificity of SEPs was superior to MEPs for hemangioma, and the sensitivity was higher with SEPs alone than with both MEP and SEP monitoring.
In some tumor types, we were unable to calculate the reliabilities of each value of EP monitoring due to the absence of a motor deterioration case (Table 6). In ependymoma, the positive and negative predictive values were higher with MEPs than SEPs, and even higher than the dual monitoring of both MEPs and SEPs. In hemangioma, SEPs showed a $100 \%$ positive predictive value, and the negative predictive value was also higher with SEPs than MEPs.

\section{DISCUSSION}

In this study, we included only patients with spinal cord tumors, with a sample size of 123 . The male to female ra- 
Table 5. Reliabilities of each combination of intraoperative MEPs and SEPs for the postoperative motor deterioration by anatomical type of spinal cord tumor

\begin{tabular}{|c|c|c|c|c|}
\hline Significant changes & Sensitivity (\%) & Specificity (\%) & PPV (\%) & NPV (\%) \\
\hline \multicolumn{5}{|l|}{ IDEM tumor $(\mathrm{n}=79)$} \\
\hline MEPs only & NA & 81.4 & NA & 98.6 \\
\hline SEPs only & 100 & 73.3 & 4.2 & 100 \\
\hline Both MEPs and SEPs & NA & 95.3 & NA & 98.8 \\
\hline Either MEPs or SEPs & 100 & 59.3 & 2.8 & 100 \\
\hline \multicolumn{5}{|l|}{ IM tumor $(\mathrm{n}=31)$} \\
\hline MEPs only & 70.8 & 81.0 & 81.0 & 70.8 \\
\hline SEPs only & 54.2 & 71.4 & 68.4 & 57.7 \\
\hline Both MEPs and SEPs & 25.0 & 95.2 & 85.7 & 52.6 \\
\hline Either MEPs or SEPs & 100 & 57.1 & 72.7 & 100 \\
\hline \multicolumn{5}{|l|}{ ED tumor $(n=11)$} \\
\hline MEPs only & 66.7 & 100 & 100 & 90.9 \\
\hline SEPs only & 66.7 & 80.0 & 50.0 & 88.9 \\
\hline Both MEPs and SEPs & 33.3 & 100 & 100 & 83.3 \\
\hline Either MEPs or SEPs & 100 & 80 & 60.0 & 100 \\
\hline
\end{tabular}

MEPs, motor evoked potentials; SEPs somatosensory evoked potentials; PPV, positive predictive value; NPV, negative predictive value; IDEM, intradural extramedullary; IM; intramedullary; ED, extradural; NA, not available.

tio in our study was similar to that in the general patient population.

Spinal cord tumors occur within or nearby the spinal cord, and account for $2 \%-4 \%$ of CNS tumors. The prevalence of certain types of tumors in our study reflected the type found in the general population. The three most common types of tumors in the United States and Japan are meningioma, schwannoma, and ependymoma, in descending order $[8,9]$. Among the cases included in this study, we also found these three to be the most common tumors, although with differing rates of abundance. Schwannoma was the most common (45.5\% of cases), followed by meningioma (18.7\%), and ependymoma (12.2\%). Differences in abundance may have occurred because we only included cases for which IONM was carried out. In particular, tumors of the spinal nerve accounted for the highest percentage of cases. IONM is more frequently carried out in function-sparing operations, where there is a benign and slow-growing tumor [10].

Our study has some strong points. First, we analyzed the spinal cord tumors by two different categories, considering the anatomical and pathologic types. Second, in each type of tumor, we calculated and compared the value for reliability. Third, we were able to verify the superiority of
SEPs in correlation with the postoperative motor deterioration in some tumor types.

We subdivided spinal cord tumors by anatomical site. Intradural extramedullary tumors are located outside the spinal cord but within the dural sheath. Intramedullary spinal cord tumors refer to a subgroup of intradural spinal tumors that arise from cells within the spinal cord, as opposed to adjacent structures, such as the nerve roots or meninges. Extradural tumors are located outside the dura mater lining [11-13].

Anatomically, MEPs monitor the corticospinal tract descending from motor cortex to the anterior horn of the spinal cord and peripheral nerve fibers [14]. The SEPs represent the function of the dorsal column-lemniscal pathway of the somatosensory system. The pathway consists of the somatosensory cortex, ventroposterior nuclei of thalamus, medial lemniscus, dorsal column, and peripheral nerve fibers [7]. Likewise, EPs' neural pathways consist of the spinal cord of the intramedullary portion and the peripheral nerve fibers of the extramedullary portion. Thus, we suggested that intraoperative neurophysiological monitoring of spinal cord tumor surgery should consider the anatomical location of tumors.

We also categorized spinal cord tumor cases by pathologic type. Some tumors like schwannoma, hemangioma, 
Table 6. Reliabilities of each combination of intraoperative MEPs and SEPs for the postoperative motor deterioration by pathologic type of spinal cord tumor

\begin{tabular}{|c|c|c|c|c|}
\hline Significant changes & Sensitivity (\%) & Specificity (\%) & PPV (\%) & NPV (\%) \\
\hline \multicolumn{5}{|l|}{ Schwannoma $(\mathrm{n}=51)$} \\
\hline MEPs only & NA & 85.2 & NA & 97.9 \\
\hline SEPs only & 100 & 74.1 & 6.7 & 100 \\
\hline Both MEPs and SEPs & NA & 96.3 & NA & 98.1 \\
\hline Either MEPs or SEPs & 100 & 63.0 & 4.8 & 100 \\
\hline \multicolumn{5}{|l|}{ Meningioma $(\mathrm{n}=25)$} \\
\hline MEPs only & NA & 80.0 & NA & 100 \\
\hline SEPs only & NA & 68.0 & NA & 100 \\
\hline Both MEPs and SEPs & NA & 96.0 & NA & 100 \\
\hline Either MEPs or SEPs & NA & 52.0 & NA & 100 \\
\hline \multicolumn{5}{|l|}{ Ependymoma (n=15) } \\
\hline MEPs only & 76.5 & 62.5 & 81.3 & 55.6 \\
\hline SEPs only & 47.1 & 62.5 & 72.7 & 35.7 \\
\hline Both MEPs and SEPs & 23.5 & 87.5 & 80.0 & 35.0 \\
\hline Either MEPs or SEPs & 100 & 37.5 & 77.3 & 100 \\
\hline \multicolumn{5}{|l|}{ Hemangioma $(\mathrm{n}=6)$} \\
\hline MEPs only & 66.7 & 75.0 & 80.0 & 60.0 \\
\hline SEPs only & 66.7 & 100 & 100 & 66.7 \\
\hline Both MEPs and SEPs & 33.3 & 100 & 100 & 50.0 \\
\hline Either MEPs or SEPs & 100 & 75.0 & 85.7 & 100 \\
\hline \multicolumn{5}{|l|}{ Neurofibroma $(\mathrm{n}=5)$} \\
\hline MEPs only & NA & 80.0 & NA & 100 \\
\hline SEPs only & NA & 80.0 & NA & 100 \\
\hline Both MEPs and SEPs & NA & 100 & NA & 100 \\
\hline Either MEPs or SEPs & NA & 60.0 & NA & 100 \\
\hline \multicolumn{5}{|l|}{ Cystic lesions (n=5) } \\
\hline MEPs only & 66.7 & 100 & 100 & 80.0 \\
\hline SEPs only & 66.7 & 100 & 100 & 80.0 \\
\hline Both MEPs and SEPs & 33.3 & 100 & 100 & 66.7 \\
\hline Either MEPs or SEPs & 100 & 100 & 100 & 100 \\
\hline \multicolumn{5}{|l|}{ Metastatic tumors $(\mathrm{n}=4)$} \\
\hline MEPs only & NA & 100 & NA & 100 \\
\hline SEPs only & NA & 100 & NA & 100 \\
\hline Both MEPs and SEPs & NA & 100 & NA & 100 \\
\hline Either MEPs or SEPs & NA & 100 & NA & 100 \\
\hline
\end{tabular}

MEPs, motor evoked potentials; SEPs somatosensory evoked potentials; PPV, positive predictive value; NPV, negative predictive value; NA, not available.

and neurofibroma are usually benign forms, while other tumors like astrocytoma, multiple myeloma, small round cell tumor are malignant forms. In the case of benign spinal cord tumors, the radical resection of the tumor is usually done for curative purposes $[15,16]$. On the other hand, in the case of malignant spinal cord tumors, tumor resection is usually performed for palliative management $[17,18]$. Moreover, the range of invasion in surgical procedure would be different according to the pathologic and anatomical type of tumor. 
For postoperative neurologic deterioration, the alert criteria for intraoperative MEP changes vary from $50 \%$ to $75 \%$ in amplitude reduction [4]. Some studies included amplitude criterion in addition to the latency criterion for SEP monitoring $[4,19,20]$. The specific alarm criteria in our spine hospital are a more than $50 \%$ reduction in MEP amplitude, and a more than $10 \%$ of N20 or P37 latency prolongation for SEPs. As the alarm criteria vary with each individual study, precise comparing of each EP value for reliability is not easy. Nevertheless, we conclude the sensitivity and specificity of MEPs for postoperative weakness was superior to that of SEPs in IONM during spine tumor surgery. Thus, we should consider why we perform intraoperative SEPs monitoring.

In this study, 2 out of 14 cases with motor deterioration showed no significant changes in MEPs, and only SEPs showed significant changes. Therefore, it seems worthwhile to perform SEP monitoring to increase the detection rate for postoperative motor deterioration, and decrease the number of false negatives [21,22]. Further, as the stimulations for MEPs make muscles contract and patients move, intraoperative MEP monitoring is usually performed only periodically when there is a major procedure. On the other hand, SEPs can be monitored relatively continuously, since the stimulations can be applied in distal area with relatively small intensity, compared to MEPs. In addition, across all cases, SEPs may be more reliable for some categories of tumor types in our study, although MEPs showed superior sensitivity and specificity in general spine surgery $[15,23]$. Although we are presenting a $100 \%$ positive predictive value of SEPs in hemangioma surgery, there may be a bias due to the small sample size. Further study with a larger sample size is needed.

SEP monitoring is known to be affected by blood flow. When the blood flow to neural tissue drops below 18 $\mathrm{mL} / 100 \mathrm{~g} / \mathrm{min}$, the SEP values start to change. Specifically, the SEP amplitudes decrease and the SEP latencies prolong. Indeed, when the blood flow to neural tissue drops below $15 \mathrm{~mL} / 100 \mathrm{~g} / \mathrm{min}$, SEP loss occurs [24,25]. However, if the SEP loss begins just before the critical threshold of persistent neurologic damage, intraoperative SEP loss can be an early warning sign for neurologic damage [21]. Thus, intraoperative SEP monitoring would be more important than MEP's in hemangioma, a highly vascularized tumor, which can lead to more blood loss during a removal operation than other types of tumors.

There are a few limitations of this study, which include the small number of patients enrolled in this study, and lack of long-term follow-up to assess neurological deteriorations. In addition, this study did not consider sensory problems as another neurologic deterioration. We only took into account SEP latencies, with no consideration of the changes in amplitudes. Further studies with a greater number of cases for each pathologic and anatomical type would bring more concise results.

In conclusion, dual IONM of both MEPs and SEPs in all cases would be ideal. However, if one must be selected due to cost or time, the selection must depend on the case at hand.

This study reconfirms the conclusion of previous studies that the highest reliability can be achieved by performing both MEPs and SEPs simultaneously. In our previous study, we reported only SEP changes predicted persistent motor deterioration in spinal cord tumor surgery, and a combined MEP/SEP monitoring showed high sensitivity and specificity in spinal deformity surgery [20]. To follow up on our previous study, we now make findings on the different sensitivities and specificities of MEPs and SEPs observed for each type of spinal cord tumor by anatomical and pathological type. This information is useful for selecting appropriate monitoring modalities for each type of surgery. Specifically, SEPs are less reliable as an overall indicator of neural deteriorations, when compared to MEPs in most types of spinal cord tumor surgeries. However, SEPs would be superior in some types of tumors, especially in hemangioma.

\section{CONFLICT OF INTEREST}

No potential conflict of interest relevant to this article was reported.

\section{ACKNOWLEDGMENTS}

This study was supported by the Je Won Research Foundation (No. 2015-31-0876).

\section{REFERENCES}

1. Lall RR, Lall RR, Hauptman JS, Munoz C, Cybulski GR, Koski T, et al. Intraoperative neurophysiological 
monitoring in spine surgery: indications, efficacy, and role of the preoperative checklist. Neurosurg Focus 2012;33:E10.

2. Malhotra NR, Shaffrey CI. Intraoperative electrophysiological monitoring in spine surgery. Spine (Phila Pa 1976) 2010;35:2167-79.

3. Scibilia A, Terranova C, Rizzo V, Raffa G, Morelli A, Esposito $\mathrm{F}$, et al. Intraoperative neurophysiological mapping and monitoring in spinal tumor surgery: sirens or indispensable tools? Neurosurg Focus 2016;41:E18.

4. Kim DG, Son YR, Park YS, Hyun SJ, Kim KJ, Jahng TA, et al. Differences in multimodality intraoperative neurophysiological monitoring changes between spinal intramedullary ependymoma and hemangioblastoma. J Clin Neurophysiol 2016;33:120-6.

5. Krammer MJ, Wolf S, Schul DB, Gerstner W, Lumenta CB. Significance of intraoperative motor function monitoring using transcranial electrical motor evoked potentials (MEP) in patients with spinal and cranial lesions near the motor pathways. Br J Neurosurg 2009; 23:48-55.

6. Sutter M, Eggspuehler A, Grob D, Jeszenszky D, Benini A, Porchet F, et al. The validity of multimodal intraoperative monitoring (MIOM) in surgery of 109 spine and spinal cord tumors. Eur Spine J 2007;16 Suppl 2: S197-208.

7. Cruccu G, Aminoff MJ, Curio G, Guerit JM, Kakigi R, Mauguiere F, et al. Recommendations for the clinical use of somatosensory-evoked potentials. Clin Neurophysiol 2008;119:1705-19.

8. Hirano K, Imagama S, Sato K, Kato F, Yukawa Y, Yoshihara $\mathrm{H}$, et al. Primary spinal cord tumors: review of 678 surgically treated patients in Japan: a multicenter study. Eur Spine J 2012;21:2019-26.

9. Duong LM, McCarthy BJ, McLendon RE, Dolecek TA, Kruchko C, Douglas LL, et al. Descriptive epidemiology of malignant and nonmalignant primary spinal cord, spinal meninges, and cauda equina tumors, United States, 2004-2007. Cancer 2012;118:4220-7.

10. Seppala MT, Haltia MJ, Sankila RJ, Jaaskelainen JE, Heiskanen O. Long-term outcome after removal of spinal schwannoma: a clinicopathological study of 187 cases. J Neurosurg 1995;83:621-6.

11. Abul-Kasim K, Thurnher MM, McKeever P, Sundgren PC. Intradural spinal tumors: current classification and MRI features. Neuroradiology 2008;50:301-14.
12. Ando K, Imagama S, Wakao N, Hirano K, Tauchi R, Muramoto A, et al. Single-stage removal of thoracic dumbbell tumors from a posterior approach only with costotransversectomy. Yonsei Med J 2012;53:611-7.

13. Koeller KK, Rosenblum RS, Morrison AL. Neoplasms of the spinal cord and filum terminale: radiologicpathologic correlation. Radiographics 2000;20:172149.

14. Macdonald DB. Intraoperative motor evoked potential monitoring: overview and update. J Clin Monit Comput 2006;20:347-77.

15. Fehlings MG, Tator $\mathrm{CH}$, Linden $\mathrm{RD}$. The relationships among the severity of spinal cord injury, motor and somatosensory evoked potentials and spinal cord blood flow. Electroencephalogr Clin Neurophysiol 1989;74:241-59.

16. Epstein FJ, Farmer JP, Freed D. Adult intramedullary spinal cord ependymomas: the result of surgery in 38 patients. J Neurosurg 1993;79:204-9.

17. Loblaw DA, Laperriere NJ. Emergency treatment of malignant extradural spinal cord compression: an evidence-based guideline. J Clin Oncol 1998;16:161324.

18. Siegal T, Siegal T. Surgical decompression of anterior and posterior malignant epidural tumors compressing the spinal cord: a prospective study. Neurosurgery 1985; 17:424-32.

19. Lee JM, Kim DH, Kim HS, Choi BK, Han IH. The applicability of intraoperative neuromonitoring in patients with preoperative motor weakness during spine surgery. Korean J Spine 2016;13:9-12.

20. Chang SH, Park YG, Kim DH, Yoon SY. Monitoring of motor and somatosensory evoked potentials during spine surgery: intraoperative changes and postoperative outcomes. Ann Rehabil Med 2016;40:470-80.

21. Toleikis JR; American Society of Neurophysiological Monitoring. Intraoperative monitoring using somatosensory evoked potentials: a position statement by the American Society of Neurophysiological Monitoring. J Clin Monit Comput 2005;19:241-58.

22. Macdonald DB, Skinner S, Shils J, Yingling C; American Society of Neurophysiological Monitoring. Intraoperative motor evoked potential monitoring: a position statement by the American Society of Neurophysiological Monitoring. Clin Neurophysiol 2013; 124:2291-316. 
23. Hilibrand AS, Schwartz DM, Sethuraman V, Vaccaro AR, Albert TJ. Comparison of transcranial electric motor and somatosensory evoked potential monitoring during cervical spine surgery. J Bone Joint Surg Am 2004;86-A:1248-53.
24. Nuwer MR. Intraoperative electroencephalography. J Clin Neurophysiol 1993;10:437-44.

25. Prior PF. EEG monitoring and evoked potentials in brain ischaemia. Br J Anaesth 1985;57:63-81. 